\title{
Size- and temperature-dependence of exciton lifetimes in CdSe quantum dots
}

\author{
C. de Mello Donegá,* M. Bode, and A. Meijerink \\ Condensed Matter and Interfaces, Debye Institute, Utrecht University, P.O. Box 80000, 3508TA Utrecht, The Netherlands \\ (Received 13 April 2006; revised manuscript received 3 July 2006; published 25 August 2006)
}

\begin{abstract}
In this work we have investigated the temperature-dependence of the band-edge photoluminescence decay of efficiently luminescing organically capped CdSe quantum dots (QDs) with diameters ranging from 1.7 to $6.3 \mathrm{~nm}$ over a broad temperature range $(1.3-300 \mathrm{~K})$. The overall trend is similar for all the investigated sizes, consisting of different temperature regimes. The low-temperature regime (below $\sim 50 \mathrm{~K}$ ) is characterized by purely radiative decay and can be modeled by a thermal distribution between a lower dark and a higher bright exciton state, with a size-dependent energy separation (viz., from 0.7 to $1.7 \mathrm{meV}$ ) and dark exciton lifetime (viz., from 0.3 to $1.4 \mu$ s for QDs ranging from $6.3 \mathrm{~nm}$ to $1.7 \mathrm{~nm}$ in diameter). Nonradiative relaxation processes become increasingly important above $\sim 50 \mathrm{~K}$ until the temperature antiquenching regime is reached, leading to a decrease in the nonradiative contributions and photoluminescence intensity recovery above $\sim 200 \mathrm{~K}$.
\end{abstract}

DOI: $10.1103 /$ PhysRevB.74.085320

PACS number(s): 78.47.+p, 78.67.Hc, 78.55.Et, 73.22.-f

\section{INTRODUCTION}

The optical properties of colloidal semiconductor nanocrystals or quantum dots (QDs) have been extensively investigated over the past decade and still attract a growing interest from both fundamental and applied viewpoints, since they reflect the remarkable size-dependent electronic structure of QDs. Potential applications include light-emitting diodes, ${ }^{1,2}$ lasers, ${ }^{1}$ photovoltaic cells, ${ }^{1,3}$ biomedical tags, ${ }^{1,4,5}$ and field-effect transistors. ${ }^{6}$ The absorption and emission transitions of semiconductor QDs are well described by a number of theoretical models, ${ }^{7-10}$ but the understanding of the exciton decay dynamics is still fragmentary, even for the prototypical colloidal QD, CdSe.

Although the band-edge luminescence lifetimes were among the first optical properties of colloidal QDs to be investigated ${ }^{11-13}$ they received much less attention than the intraband relaxation dynamics (exciton cooling), ${ }^{14-17}$ and it was only recently that new investigations on the subject appeared. ${ }^{18-30}$ The initial studies ${ }^{11,12}$ revealed that the lowtemperature exciton lifetime of CdSe QDs dramatically differs from that of the bulk counterpart, being orders of magnitude longer (viz., microseconds ${ }^{11,12}$ instead of $\sim 600 \mathrm{ps}$ Ref. 31). Furthermore, fluorescence line-narrowing (FLN) experiments on CdSe QDs (Ref. 12) demonstrated that both the resonant Stokes shift $\Delta_{S T}$ and the Huang-Rhys parameter $S$ (i.e., the intensity ratio between the phonon replicas and the zero-phonon line) increase upon cooling from 10 to $1.75 \mathrm{~K}$, while the homogeneous linewidth $\sigma$ remains virtually constant. Size-dependent studies demonstrated that $\sigma, \Delta_{S T}, S$, the radiative lifetimes and the temperaturedependence of $S$ decrease dramatically with increasing QD size $\left(2.3-8.3 \mathrm{~nm}\right.$ diameter). ${ }^{12}$ These observations were initially ascribed to surface localization of the photogenerated hole, ${ }^{11,12}$ which would give rise to two emitting surface states: a lower one with a small oscillator strength (strongly localized) and an upper one with a larger oscillator strength (mostly delocalized). However, a subsequent investigation carried out by the same group, ${ }^{32}$ using transient differential absorption (TDA) and gated FLN spectroscopy, cast doubt on the surface-based interpretation, which was shown to be inconsistent with the strong similarity observed between the lowest energy bleach peak in the TDA spectra and the zerophonon line in the FLN spectra. Further experiments [viz., size-dependent FLN spectra at $10 \mathrm{~K},{ }^{7,13}$ magnetic field dependence of FLN spectra and lifetimes at $1.7 \mathrm{~K},{ }^{7,13}$ FLN spectroscopy on surface modified CdSe and CdSe/ZnS QDs (Ref. 33)] and theoretical modeling ${ }^{7,13}$ established that the energetics and dynamics of the exciton photoluminescence could be understood in terms of the intrinsic band-edge exciton fine structure, without the need to invoke surface effects, which were then deemed responsible solely for the nonradiative relaxation pathways and defect related luminescence. ${ }^{7,33}$

The asymmetric crystal field in CdSe (wurtzite structure) splits the lowest exciton state, $1 S_{(e)} 1 S_{3 / 2(h)}$, into two fourfold degenerate states (the $A$ and $B$ excitons in bulk CdSe). ${ }^{7}$ Crystal shape anisotropy and electron-hole exchange interaction partially lift the degeneracy of the $A$ and $B$ excitons, yielding five levels (Fig. 1), two of them optically passive in the electric-dipole approximation (i.e., their total angular momentum projection is such that transitions to or from these states are electric-dipole forbidden). ${ }^{7}$ The energy levels presented in Fig. 1 are labeled according to their total angular momentum projection along the wurtzite hexagonal axis. ${ }^{7}$ Optical transitions are electric-dipole forbidden for the $E_{ \pm 2}$ and the $E_{0}^{L}$ levels and allowed for the $E_{ \pm 1}^{L}, E_{ \pm 1}^{U}$, and $E_{0}^{U}$ levels, and therefore, these levels are usually referred to as "dark" and "bright" exciton states, respectively. This model is strongly supported by the insensitivity of the absorption, band-edge photoluminescence and FLN spectra to the properties of the QD surface. ${ }^{33}$ Further it has successfully explained a large body of experimental results: the sizedependence of the fine structure observed in excitation ${ }^{34}$ and emission $^{7,13,34}$ and of the homogeneous and inhomogeneous Stokes shift, ${ }^{7,13}$ and the magnetic field dependence of the low-temperature exciton lifetimes and fine structure (viz., shortening of the lifetimes and an increase of the zerophonon line intensity with increasing magnetic field $\left.{ }^{7,13}\right)$. The anomalously long low-temperature exciton lifetimes ${ }^{7,13}$ are 

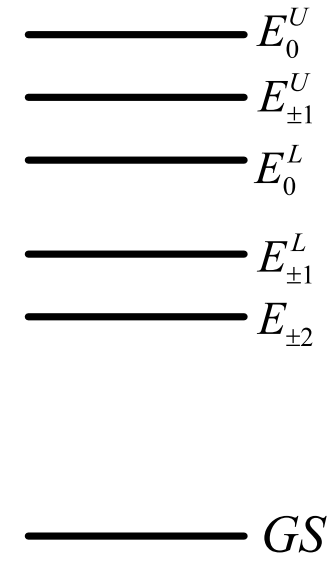

FIG. 1. Schematic energy level scheme showing the band-edge exciton fine structure for CdSe QDs. Following Efros and coworkers (Ref. 7), the levels are labeled according to their total angular momentum projection along the wurtzite hexagonal axis $(G S=$ ground state $)$.

also qualitatively explained, since the lowest exciton state is optically passive (the so-called dark exciton), and is followed by a higher-lying optically active state (bright exciton). ${ }^{7}$ At low temperatures (below $\sim 2 \mathrm{~K}$ ) the luminescence originates primarily from the dark exciton, leading to long exciton lifetimes (viz., microseconds). ${ }^{11,12}$ The energy gap between these two states (the dark-bright gap) was taken to be the resonant Stokes shift and shown to increase with decreasing QD size (viz., $1.2-19 \mathrm{meV}$ from 11.5 to $2.4 \mathrm{~nm}$ diameter ${ }^{7,13}$ ), in agreement with the theoretically predicted enhancement of the electron-hole exchange interaction with increasing quantum confinement. ${ }^{7,13}$

Although the temperature- and size-dependence of the exciton lifetimes of QDs would provide a direct way to determine the dark-bright energy gap, they were not systematically investigated until recently, when two groups independently reported on the exciton lifetimes of $\mathrm{CdSe} / \mathrm{ZnS}$ core-shell QDs at several temperatures. ${ }^{24,25}$ The reported results and interpretations, however, are not fully consistent with each other. ${ }^{24,25}$ Crooker et al. $^{24}$ investigated QD ensembles (average diameters: 2.6, 3.7, and $4.2 \mathrm{~nm}$, quantum yield: $35 \%$ at $300 \mathrm{~K}$ ) over a wide temperature range $(0.38-300 \mathrm{~K})$ and concluded that there is an intrinsic temperature independent radiative limit of $\sim 1 \mu$ s below $2 \mathrm{~K}$, which depends only weakly on QD size and surface passivation, and that above $2 \mathrm{~K}$ the exciton lifetimes are governed by equilibrium between dark and bright excitons, but with two different energy gaps: a smaller one $(\sim 1 \mathrm{meV}$, size independent) dominating below $20 \mathrm{~K}$, and a larger one, size dependent (values not reported), governing the dynamics above $20 \mathrm{~K}$. The larger energy gap was ascribed to the intrinsic band-edge exciton fine structure, while a weak exchange interaction with surface dangling bonds was proposed to account for the $\sim 1 \mathrm{meV}$ activation energy. ${ }^{24}$ In contrast, the temperature-dependence observed by Labeau et al. ${ }^{25}$ for single $\mathrm{CdSe} / \mathrm{ZnS}$ QDs (average ensemble diameters: $3.0,3.8$, and $11.2 \mathrm{~nm}$ ) in the $2-140 \mathrm{~K}$ range was satisfactorily explained by a three-level model with a single sizedependent dark-bright energy gap (1.3-3.8 meV for $3.8 \mathrm{~nm}$
QDs, 3.6-7 meV for $3.0 \mathrm{~nm}$ QDs, and $2 \mathrm{meV}$ for $11.2 \mathrm{~nm}$ QDs). It is not clear whether the inconsistency between the results of Crooker et al. ${ }^{24}$ and Labeau et al. ${ }^{25}$ can be explained by ensemble effects or different degrees of nonradiative contribution and sample inhomogeneities.

In this work we intend to resolve the contradiction mentioned above, while providing a better understanding of the size- and temperature-dependence of exciton lifetimes in CdSe QDs. To this end we investigate the temperaturedependence of the band-edge luminescence decay over a broad temperature range $(1.3-300 \mathrm{~K})$ for a series of efficiently luminescing organically capped CdSe QDs over a wide size range (diameters ranging from 1.7 to $6.3 \mathrm{~nm}$ ). We note that highly efficient organically capped CdSe QDs offer the best possible system to investigate the intrinsic exciton dynamics, since they have a nearly defect free surface, as evidenced by their high photoluminescence quantum yield (as high as $85 \%$ at room temperature ${ }^{21,35}$ ), absence of defect related luminescence, and (nearly) single exponential exciton decays even at room temperature. ${ }^{27,28}$ Core-shell QDs, albeit rather efficient, have the potential disadvantage that the coreshell interface itself might act as a perturbation, due to either interfacial strain ${ }^{36}$ or compositional fluctuations. To probe whether this hypothesis is justified we have also investigated a sample of $\mathrm{CdSe} / \mathrm{ZnSe} / \mathrm{ZnS}$ core-shell-shell QDs for comparison. $\mathrm{ZnSe} / \mathrm{ZnS}$ double shells were preferred over single material shells because they provide a good surface passivation while minimizing the core-shell interfacial strain. ${ }^{36,37}$ Furthermore, a $\mathrm{CdSe} / \mathrm{ZnSe}$ core-shell interface is probably closer to the surface of the organically capped CdSe QDs investigated in this work than $\mathrm{CdSe} / \mathrm{CdS}$ or $\mathrm{CdSe} / \mathrm{ZnS}$ interfaces.

\section{EXPERIMENT}

The CdSe quantum dot samples investigated in this work were grown by hot injection of organometallic precursors into a coordinating solvent mixture, following the method reported by de Mello Donegá et al. ${ }^{21}$ This method yields high-quality organically capped CdSe nanocrystals, which present high photoluminescence quantum yields (30-90\% at room temperature), no defect related luminescence, and small size polydispersity (4-10\%) in the $1.7-7 \mathrm{~nm}$ diameter range.

The preparation consists of injecting a precursor solution (0.79 $\mathrm{g} \mathrm{Se}$ and $0.28 \mathrm{~g} \mathrm{Cd}(\mathrm{Me})_{2}$ in $10 \mathrm{~mL}$ trioctylphosphine, TOP) into a hot $\left(300{ }^{\circ} \mathrm{C}\right)$ coordinating solvent mixture $(20 \mathrm{~g}$ trioctylphosphineoxide, TOPO, and $10 \mathrm{~g}$ Hexadecylamine, HDA). The nanocrystals are then grown at $240{ }^{\circ} \mathrm{C}$, yielding CdSe QDs with diameters ranging from 2.7 to $3.9 \mathrm{~nm}$, depending on the growth duration (15-90 $\mathrm{min})$. To grow QDs larger than $3.9 \mathrm{~nm}$ additional precursors are slowly added while keeping the temperature constant. QDs smaller than $2.7 \mathrm{~nm}$ require a modified procedure, with lower injection and growth temperatures. Further, nucleation and growth are carried out in different coordinating solvents. Nucleation is achieved by injecting a precursor solution $[0.79 \mathrm{~g}$ Se and $0.87 \mathrm{~g} \mathrm{Cd}(\mathrm{Me})_{2}$ in $10 \mathrm{~mL}$ TOP] into $10 \mathrm{~mL} 2 \mathrm{M}$ TOP-Se at $100{ }^{\circ} \mathrm{C}$ followed by dilution and cooling with $10 \mathrm{~mL}$ TOP at 
room temperature. This process yields a solution of $1.2 \mathrm{~nm}$ CdSe nanocrystals in TOP, which are subsequently grown to either $1.7 \mathrm{~nm}$ in TOP-dodecylamine at $150{ }^{\circ} \mathrm{C}$ or to $1.9-2.5 \mathrm{~nm}$ in TOPO-HDA-TOP at temperatures between $150{ }^{\circ} \mathrm{C}$ and $210{ }^{\circ} \mathrm{C}$. CdSe QDs overcoated by a $\mathrm{ZnSe} / \mathrm{ZnS}$ double shell (2 ML ZnSe and $1 \mathrm{ML} Z \mathrm{ZnS})$ are prepared by a one-pot method, developed by combining and adapting a number of methods available in the literature. ${ }^{36-39}$ To grow the $\mathrm{ZnSe}$ shell a $\mathrm{Zn}(\mathrm{Et})_{2}$ solution in TOP $(0.12 \mathrm{~g}$ in $4 \mathrm{~mL}$ TOP) is slowly added $(0.05 \mathrm{~mL} / \mathrm{s})$ to a solution of CdSe QDs in TOPO-HDA-TOP (6 $\mathrm{g}$ crude reaction mixture, $8 \mathrm{~g}$ TOPO, $4 \mathrm{~g} \mathrm{HDA})$ at $150{ }^{\circ} \mathrm{C}$. After $20 \mathrm{~min}$ annealing at $150{ }^{\circ} \mathrm{C}$ a solution of $0.16 \mathrm{~g}$ elemental sulphur in $3 \mathrm{~mL}$ TOP is added, followed by slow addition of $\mathrm{Zn}(\mathrm{Et})_{2}(0.1 \mathrm{~g}$ in 4 $\mathrm{mL}$ TOP). The temperature is then raised to $170{ }^{\circ} \mathrm{C}$ over 15 min and kept constant for $10 \mathrm{~min}$, after which the reaction mixture is quickly cooled down to $100{ }^{\circ} \mathrm{C}$ and annealed for $1 \mathrm{~h}$. All syntheses were carried out in a glove box under argon (less than 2 ppm $\mathrm{O}_{2}$ and $\mathrm{H}_{2} \mathrm{O}$ ). In order to minimize reabsorption and avoid energy transfer between the QDs all optical measurements were carried out on samples with a low optical density ( $\leqslant 0.05$ at the emission maximum, $\sim 0.2$ at $400 \mathrm{~nm}$, corresponding to QD concentrations in the $0.1 \mu \mathrm{M}$ range $\left.{ }^{40,41}\right)$. All samples were prepared by directly dissolving the crude reaction mixture in anhydrous toluene under argon. Photoluminescence (PL) spectra were acquired upon excitation at $406 \mathrm{~nm}$, using as excitation source, either a Pico Quant PDL 800-B laser or a $450 \mathrm{~W}$ Xe lamp combined with a $0.22 \mathrm{~m}$ monochromator. The emitted light was collimated into an optical fiber cable leading to a $0.3 \mathrm{~m}$ monochromator (Acton Pro SP-300i, 150 lines/mm grating, blazed at $500 \mathrm{~nm}$ ) and detected by a liquid $\mathrm{N}_{2}$-cooled Princeton Instruments charge-coupled device camera $(1024 \times 256$ pixels $)$. UV-visible absorption spectra were recorded on a Perkin-Elmer Lambda $16 \mathrm{UV} / \mathrm{V}$ is spectrophotometer.

The excitonic transition energy, derived from the maximum of the first exciton absorption peak, was used to estimate the average nanocrystal diameter by fitting to an empirical calibration curve correlating the exciton peak energy with the nanocrystal size, constructed from experimental data published by several independent groups. ${ }^{40-45}$ The accuracy and reliability of this calibration curve was checked by determining the nanocrystal sizes of several samples (2.5-12 $\mathrm{nm}$ size range) by transmission electron microscopy (TEM) (FEI TECNAI G ${ }^{2}$ T20F $-200 \mathrm{KeV}$ ). The TEM sizes are in good agreement $( \pm 10 \%)$ with the values estimated from the exciton absorption peaks. Since the nanocrystals are prolate (aspect ratio: $1.3 \pm 0.2$ ) the shorter axis dimension is taken as the diameter. PL quantum yields (QY) were obtained by comparison with a standard dye, following the method reported in Ref. 21.

PL decay curves were obtained by using a Pico Quant PDL 800-B laser as the excitation source $\left(\lambda_{\text {exc }}=406 \mathrm{~nm}\right.$, 55 ps pulse width). The repetition rates were controlled by using either an external pulse generator (Stanford DG535, 0.2-2.0 MHz) or the laser driver internal clock $(2.5-40 \mathrm{MHz})$. Very low excitation fluences were used $\left(<0.5 \mathrm{~nJ} / \mathrm{cm}^{2}\right)$ to avoid multiexciton formation. A combina- tion of iris and neutral density filters was used to attenuate the laser power as needed. The PL was filtered through a crossed polarizer and a combination of suitable optical cutoff filters to eliminate scattered laser light, dispersed by a $0.1 \mathrm{~m}$ monochromator (1350 lines $/ \mathrm{mm}$ grating, blazed at $500 \mathrm{~nm}$ ) and detected by a fast Hamamatsu photomultiplier tube (PMT) (H5738P-01). The PMT signal was amplified by an inverting preamplifier (PAM-102-T-PicoQuant $\mathrm{GmbH}$ ) and used as the start input for a Time Harp 200 multichannel computer card (PicoQuant $\mathrm{GmbH}$ ), synchronized with the laser pulse via the stop input. The decay curves were obtained by time correlated single-photon counting via time-toamplitude conversion, while monitoring the PL peak wavelength. The ratio of stop to start pulses was kept low (below $0.04)$ to assure good statistics. The sample was contained in a sealed quartz cuvette (optical path: $3 \mathrm{~mm}$ ) and mounted in a continuous He-flow cryostat (Optistat CF Oxford Instruments) allowing for measurements down to $1.2 \mathrm{~K}$. The raw decay data were iteratively deconvoluted from the instrument response function (IRF) and fitted to decay functions using a Simplex minimization algorithm implemented in PicoQuant FluoFit 3.3. To allow consistent comparison between different samples and temperatures while ensuring that the obtained decay constants were statistically valid and representative of the whole QD ensemble the decay data were fit from $t=100 \%$ to $2 \% I_{0}$ using only three decay functions: biexponential, single exponential, or Gaussian distribution. The fit quality was assessed by two criteria: reduced $\chi^{2}$ $(\approx 1)$ and the weighed residuals' autocorrelation function (random distribution of small values). The IRF was 700 ps (full width at half maximum) at $2.5 \mathrm{MHz}$ repetition rate.

\section{RESULTS AND DISCUSSION}

The room temperature (RT) band-edge PL spectra of the investigated CdSe QD samples consist of a narrow band with a maximum ranging from 485 to $658 \mathrm{~nm}$, depending on the QD diameter $(1.7-6.3 \mathrm{~nm})$. The full width at half maximum (FWHM) varies between 90 and $150 \mathrm{meV}$ for all samples. Defect related PL is observed only for the $1.7 \mathrm{~nm}$ QDs (a broad band $\sim 0.6 \mathrm{eV}$ redshifted with respect to band-edge PL) and contributes $20 \%$ to the integrated PL intensity. The RT band-edge PL QY are 40-60\% for QDs in the $2.8-5.0 \mathrm{~nm}$ diameter range, $30 \%$ for the 2.0 and $6.3 \mathrm{~nm}$ samples, and $20 \%$ for the $1.7 \mathrm{~nm}$ diameter CdSe QDs. The PL QY of the CdSe/ZnSe/ZnS core-shell-shell QDs is 55\%, which is slightly higher than the PL QY of the bare $2.8 \mathrm{~nm}$ cores (viz., 40\%), attesting to the good surface quality of the organically capped CdSe QDs investigated in this work. CdSe QDs (2.5 nm diameter) with a low RT PL QY (viz., $5 \%)$ were also investigated for comparison.

The PL spectra at low temperatures are essentially the same as at RT, but the FWHM decreases to $80-110 \mathrm{meV}$ and the exciton PL peak blueshifts by $40-50 \mathrm{meV}$ as the temperature decreases from RT to $4 \mathrm{~K}$. The blueshift of the exciton peak energy can be ascribed to the temperaturedependence of the band gap, and has been shown to be similar to that of bulk CdSe, ${ }^{46}$ which amounts to $90 \mathrm{meV} .{ }^{47}$ The exciton PL linewidth is determined by both inhomoge- 

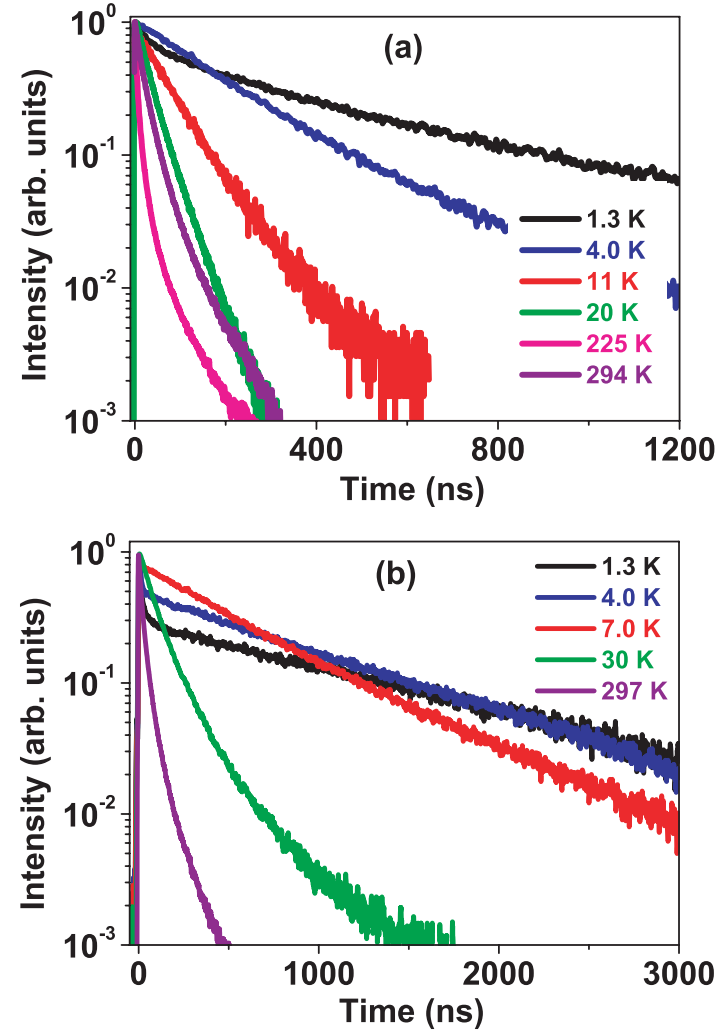

FIG. 2. (Color) Photoluminescence decay curves for (a) $4.3 \mathrm{~nm}$ diameter and (b) $1.7 \mathrm{~nm}$ diameter CdSe QDs at the temperatures indicated $\left[\lambda_{\text {excitation }}: 406 \mathrm{~nm}, \lambda_{\text {emission }}: 609 \mathrm{~nm}\right.$ and $483 \mathrm{~nm}$, for (a) and (b), respectively].

neous broadening, which is mostly due to size and shape inhomogeneities within the QD ensemble, and homogeneous broadening, which results from exciton-phonon interactions and is therefore temperature dependent. A detailed analysis of the temperature dependence of the exciton line broadening can provide information on the exciton-phonon coupling in QDs. ${ }^{46}$ However, measurements performed on QD ensembles are not ideally suited for such an analysis because the linewidths are mostly due to inhomogeneous broadening, particularly at low temperatures.

The temperature-dependence of the PL decay curves is quite pronounced, especially below $\sim 50 \mathrm{~K}$. As representative examples, Fig. 2 shows band-edge PL decay curves of 1.7 and $4.3 \mathrm{~nm}$ CdSe QDs at selected temperatures between 1.3 and $300 \mathrm{~K}$. Figure 3 shows the exciton lifetimes as a function of temperature for all the investigated samples. The temperature-dependence of the integrated PL intensities (obtained by integrating the decay curves) for the $4.3 \mathrm{~nm} \mathrm{CdSe}$ QDs sample is presented in Fig. 4. The overall trends are similar for all the investigated QD sizes. The combined analysis of the decay curves and the integrated intensities allows three temperature regimes to be discerned.

The low-temperature regime (up to $\sim 50 \mathrm{~K}$, for all sizes) is characterized by nearly constant PL intensity and single exponential decays, implying that the exciton decay rates correspond to radiative decay in this temperature range ("radiative regime," I in Fig. 4). At $1.3 \mathrm{~K}$ the PL decay curves are biexponential for all QD sizes (and samples, regardless of

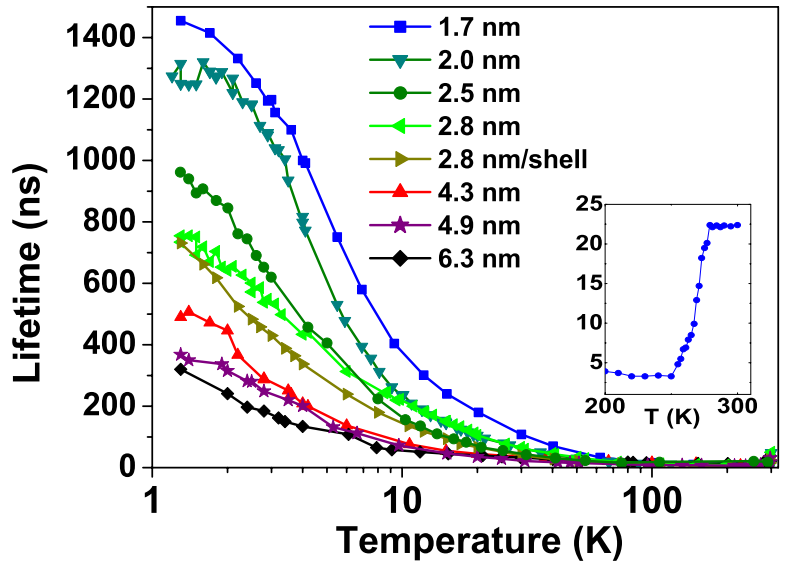

FIG. 3. (Color online) Temperature-dependence of the exciton lifetimes of organically capped CdSe QDs with diameters ranging from 1.7 to $6.3 \mathrm{~nm}$. Results for a core-shell system (viz., $\mathrm{CdSe} / \mathrm{ZnSe} / \mathrm{ZnS}$ ) are also included. Temperature is given on a log scale. The inset shows the region above $200 \mathrm{~K}$ (linear scale) for the $4.3 \mathrm{~nm}$ sample, as a representative example.

the RT QY) (see Fig. 2 for representative examples). The fast component in the biexponential decay curves is ascribed to bright exciton decay prior thermalization between the lower (dark exciton) and the upper (bright exciton) emitting states. As the temperature is raised the fast component gradually disappears and the slow component becomes faster until a single exponential behavior is reached at $\sim 10 \mathrm{~K}$. In the case of biexponential decays, Fig. 3 presents the lifetime for the slow exponential component. The decay curves remain exponential and the decay rates become increasingly faster up to $\sim 50 \mathrm{~K}$, when the PL intensity starts to decrease (see, e.g., Fig. 4) and the decays become faster and nonexponential with increasing temperature (see, e.g., Fig. 2), signaling thermally activated exciton trapping and nonradiative decay (the "quenching regime," II in Fig. 4). The lifetimes presented in Fig. 3 for this temperature range are extracted by modeling the decay curves as log-Gaussian distributions of rate

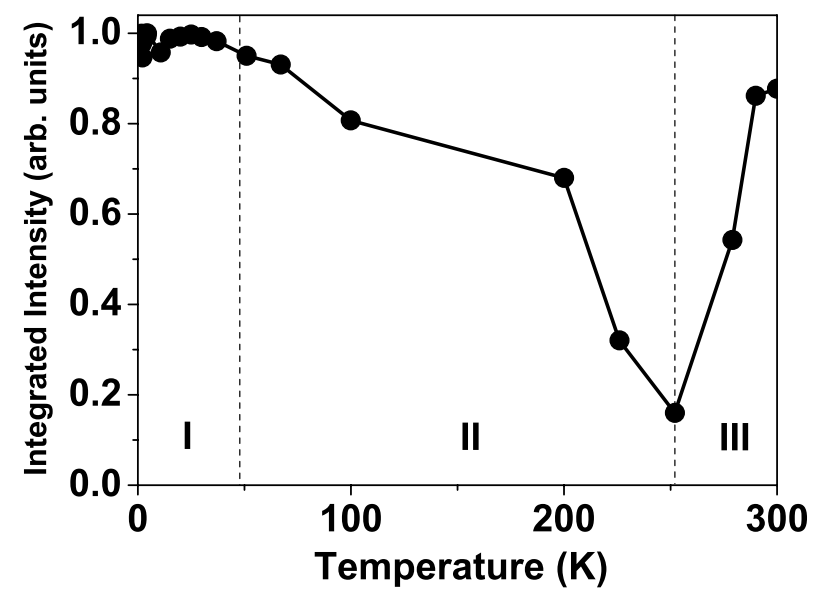

FIG. 4. Temperature-dependence of the integrated photoluminescence intensities (integrated decay curves) for $4.3 \mathrm{~nm}$ diameter CdSe QDs (I=radiative regime; II=quenching regime; $\mathrm{III}=$ temperature antiquenching regime). 
constants, which provides a realistic description of systems dominated by trapping dynamics, since the trap depths will vary across the QD ensemble and also fluctuate in time for individual QDs. ${ }^{26}$ The lifetimes determined from the exponential tails of the decay curves $\left(I<5-10 \% I_{0}\right)$ reflect the radiative exciton recombination and are observed to remain nearly constant above $\sim 50 \mathrm{~K}$, in agreement with the observations reported in Ref. 24, where the exciton lifetimes were determined from single exponential fits to the tail of the decay curves $\left(I<5 \% I_{0}\right)$ at all temperatures.

The onset of the quenching regime does not appear to be size-dependent, beginning at $\sim 50 \mathrm{~K}$ for all efficient samples (RT QY $\geqslant 20 \%$ ), regardless of QD size or the nature of the surface passivation (i.e., TOPO-TOPSe-HDA organic capping or a $\mathrm{ZnSe} / \mathrm{ZnS}$ shell). A poorer surface quality, as judged by a low RT QY, does not lead to a significantly lower onset temperature for quenching but rather to a more pronounced decrease in the lifetimes above the onset temperature (see, e.g., the $2.5 \mathrm{~nm}$ sample in Fig. 3, with RT $\mathrm{QY}=5 \%$ ). Above $\sim 250 \mathrm{~K}$ the PL intensities begin to recover (Fig. 4) and the lifetimes start to increase again (see inset in Fig. 3 for an example, and also Ref. 30), reaching a (nearly) single exponential decay at room temperature (Fig. 2 and Ref. 30). Temperature quenching of the photoluminescence of QDs is a commonly observed phenomenon, both in colloidal suspensions or in solvent-free systems such as QDs embedded in polymeric matrices and QD solids, and is ascribed to thermally activated carrier trapping. ${ }^{24,46,48}$ The thermally induced luminescence recovery observed here (Figs. $2-4)$ is thus highly remarkable. This unusual behavior (dubbed "luminescence temperature antiquenching"LTAQ) has been investigated in detail by our group 29,30 and shown to be strongly dependent on the surface ligands. For colloidal suspensions of CdSe QDs capped by linear alkylamines the onset of the PL recovery shifts to higher temperatures as the alkyl chain length increases from $\mathrm{C} 6$ to $\mathrm{C} 18$, consistent with a phase transition in the capping layer which allows surface relaxation and/or reconstruction to take place, thereby moving surface states away from the band gap. ${ }^{30}$ This transition is probably analogous to the "locked-tounlocked rotator" phase transition observed in selfassembled monolayers of alkylamines, by which the chains recover precessional mobility, albeit remaining ordered. ${ }^{30} \mathrm{We}$ note that the LTAQ transition is not observed for colloidal suspensions of $\mathrm{ZnS}$ (or $\mathrm{ZnSe} / \mathrm{ZnS}$ ) overcoated CdSe QDs, showing that the surface structure of core-shell QDs does not observably affect the optical properties of the CdSe core, as expected considering the superior photochemical stability of core-shell QDs with respect to organically capped bare QDs. ${ }^{36-39}$

The most dramatic size- and temperature- dependence is observed below $\sim 50 \mathrm{~K}$ (regime I in Fig. 4), implying that the exciton radiative lifetime itself is changing. The temperature dependence of the exciton lifetimes in the radiative regime (regime I) can be modeled by a three-level system, assuming a thermal equilibrium between two emitting states, ${ }^{49}$ the lower one presenting a longer lifetime (dark exciton) than the upper one (bright exciton):

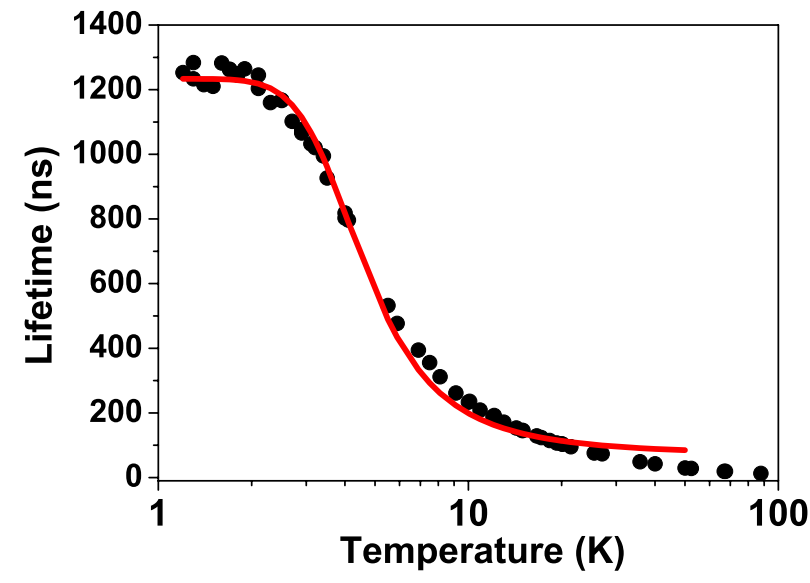

FIG. 5. (Color online) Three-level fit (solid line) to the experimental temperature dependence of the exciton lifetimes of $2.0 \mathrm{~nm}$ diameter CdSe QDs using Eq. (1) and $\tau_{\text {dark }}=1235 \mathrm{~ns}$; $\tau_{\text {bright }}=40 \mathrm{~ns}$; and $\Delta E=1.39 \mathrm{meV}$, in the temperature range $1.3-50 \mathrm{~K}$.

$$
\frac{1}{\tau}=\frac{1}{\tau_{\text {dark }}}\left(\frac{e^{\Delta E / k T}}{1+e^{\Delta E / k T}}\right)+\frac{1}{\tau_{\text {bright }}}\left(\frac{1}{1+e^{\Delta E / k T}}\right),
$$

where $\tau$ is the observed radiative lifetime, $\tau_{\text {dark }}$ and $\tau_{\text {bright }}$ are the dark and the bright exciton lifetimes, respectively, and $\Delta E$ gives the energy gap between the two emitting states. The energy gap between the bright exciton and the subsequent levels is assumed to be much larger than the darkbright energy gap $\Delta E$. Despite its simplicity, this model provides a reasonable fit to the temperature dependence of the exciton lifetimes below $\sim 40 \mathrm{~K}$ (see Fig. 5, for an example). The small deviation observed between the three-level fit and the experimental temperature-dependence below $40 \mathrm{~K}$ may be due to size and shape inhomogeneities within the QD ensemble and/or to the fact that higher lying levels may also participate, so that more than three levels would be needed to quantitatively describe the observed decay dynamics.

A clear discrepancy between the fitted curve and the data is observed as the temperature increases above $40 \mathrm{~K}$ (Fig. 5), consistent with the onset of the quenching regime. The experimentally observed lifetimes are faster than the calculated (radiative) decay rates due to nonradiative transitions. It is interesting to note that if the values obtained from the fit up to $40 \mathrm{~K}$ are extrapolated to RT, a good agreement is obtained for all lifetimes above the LTAQ transition temperature. ${ }^{30}$ This clearly shows that the temperature dependence of the exciton lifetimes above $\sim 40 \mathrm{~K}$ (regimes II and III in Fig. 4) can be ascribed to an increase (quenching regime) or decrease (LTAQ regime) of the nonradiative contributions to the decay rates. We note that single exponential decays, reflecting purely radiative exciton recombination, can be observed even for QD samples with ensemble RT QYs well below $100 \%$, provided that the ensemble consists of a distribution of very efficient QDs (with near unity QY) and nearly nonluminescent (dark) QDs. ${ }^{25-27,50}$ Coexistence of bright and dark QDs in the same ensemble has been demonstrated by $\mathrm{PL}$ and decay measurements on single QDs. ${ }^{25,26,50}$ 

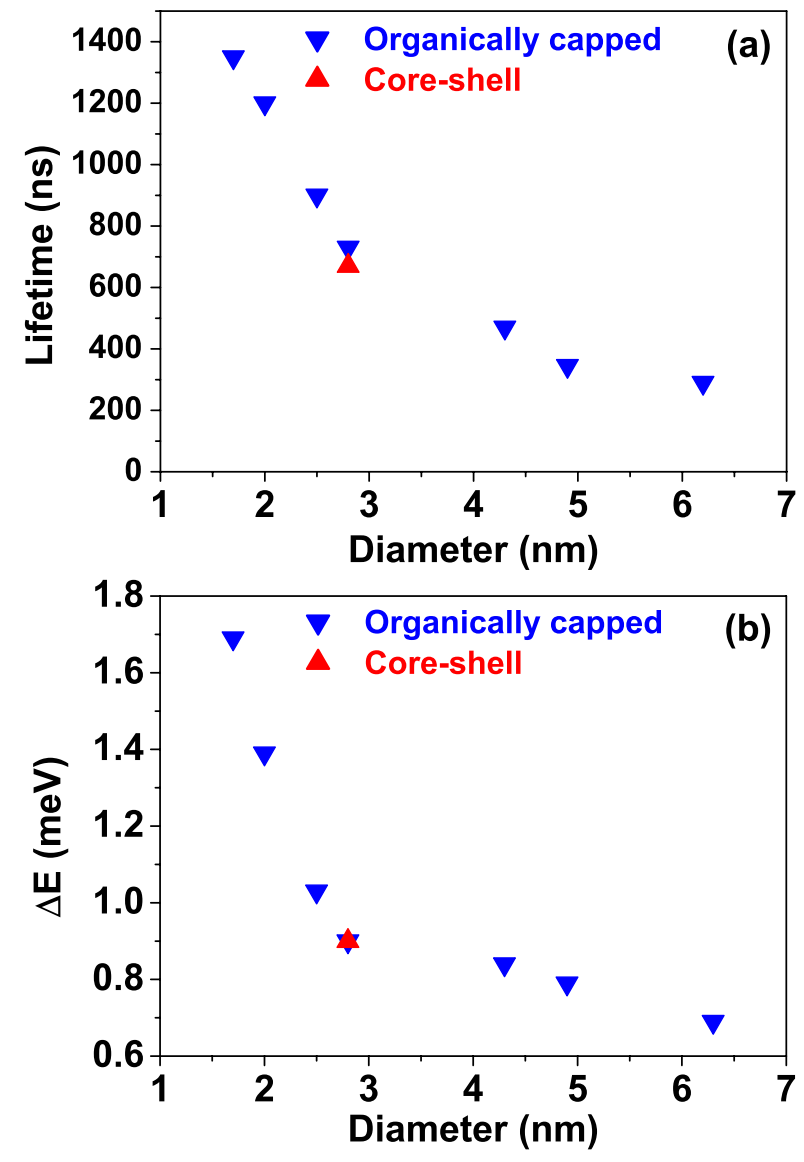

FIG. 6. (Color online) (a) Dark exciton radiative lifetimes $\left(\tau_{\text {dark }}\right)$ and (b) dark-bright energy gap $(\Delta E)$ obtained from fitting Eq. (1) to the experimental temperature-dependence of the exciton lifetimes of organically capped CdSe QDs with diameters ranging from 1.7 to $6.3 \mathrm{~nm}$. Results for a sample of $\mathrm{CdSe} / \mathrm{ZnSe} / \mathrm{ZnS}$ core-shell QDs are also included.

The results presented here clearly show that the temperature-dependence of the exciton lifetimes in the radiative regime (i.e., below $\sim 50 \mathrm{~K}$, regime I in Fig. 4) can be modeled only if both the dark exciton lifetime $\left(\tau_{\text {dark }}\right)$ and the dark-bright energy gap $(\Delta E)$ are size dependent (Fig. 6). To understand this size-dependence the nature of the dark and bright emitting states has to be considered. We will first analyze whether the intrinsic CdSe band-edge exciton fine structure $^{7,9,10}$ can explain our observations and subsequently discuss alternative explanations, such as surface based models. ${ }^{12,24,51,52}$

The size-dependent trend observed in Fig. 6(b) for the dark-bright energy gap $(\Delta E)$ is qualitatively similar to those reported in the literature, ${ }^{7,13}$ in agreement with the expected enhancement of the electron-hole exchange interaction for decreasing QD sizes, ${ }^{7,9,13}$ but the absolute values (0.7-1.7 $\mathrm{meV}$ in the $6.3-1.7 \mathrm{~nm}$ diameter range) are significantly smaller than those extracted from FLN measurements (1.2-19 meV from 11.5 to $2.4 \mathrm{~nm}$ diameter $\left.{ }^{7,13}\right)$ or theoretically modeled $\left(0.2-13.5 \mathrm{meV}\right.$ from 10 to $2 \mathrm{~nm}$ diameter; ${ }^{7}$ $3-17 \mathrm{meV}$ from 7.5 to $1.7 \mathrm{~nm}$ diameter; $94.4-16 \mathrm{meV}$ from 3.7 to $1.9 \mathrm{~nm}$ diameter ${ }^{10}$ ).

We note that taking the resonant Stokes shift $\left(\Delta_{S T}\right)$ observed in FLN experiments to be the energy gap between the bright and dark excitons (the $E_{ \pm 1}^{L}$ and $E_{ \pm 2}$ levels in Fig. 1, respectively) implies the assumption that $\Delta_{S T}$ is solely due to the $E_{ \pm 1}^{L} \rightarrow E_{ \pm 2}$ relaxation, thus neglecting any internal relaxation of the exciton states (e.g., dephasing by coupling to phonons). Considering that the low-temperature $(10 \mathrm{~K})$ homogeneous linewidth $(\sigma)$ and $\Delta_{S T}$ are comparable [e.g., $\sigma=17 \mathrm{meV}$ and $\Delta_{S T}=18 \mathrm{meV}$ for $2.3 \mathrm{~nm}$ CdSe QDs; $\sigma=4 \mathrm{meV}$, and $\Delta_{S T}=1 \mathrm{meV}$ for $8.3 \mathrm{~nm}$ QDs (Ref. 12)], we argue that this assumption is not justified and therefore the $\Delta_{S T}$ values obtained from FLN spectra will not correspond to the exact energy separation between the $E_{ \pm 1}^{L}$ and the $E_{ \pm 2}$ exciton levels $(\Delta E)$. In fact, this implies that $\Delta_{S T}$ will be systematically larger than $\Delta E$, which is consistent with the observation that the $\Delta_{S T}$ values for QDs larger than $\sim 7 \mathrm{~nm}$ [1.2-2 meV (Refs. 7 and 13)] are one order of magnitude larger than the bulk value [viz., $0.13 \mathrm{meV}$ (Refs. 7 and 9)].

The theoretical approach proposed by Efros et al. ${ }^{7}$ to model the band-edge exciton fine structure in QDs is based on the effective mass approximation (EMA), treating crystalfield effects, shape asymmetry, and exchange interactions as perturbations that split the eightfold degenerate band-edge exciton state $\left(1 S_{(e)} 1 S_{3 / 2(h)}\right)$ into five levels (Fig. 1). The asymmetric wurtzite crystal field (c.f.) lifts the hole state degeneracy, splitting it into two twofold degenerate states $\left(1 S_{3 / 2(h)}\right.$ and $\left.1 S_{1 / 2(h)}\right)$. Shape asymmetry also lifts hole state degeneracy, but is size dependent and may lead to contributions with signs opposite to the c.f. induced splitting, therefore changing the order of the levels for sufficiently small prolate nanocrystals. ${ }^{7}$ A deviation from spherical symmetry is modeled by taking the nanocrystal to be an ellipsoid, which can be prolate or oblate. The electron-hole exchange interaction mixes different electron and hole spin states, thereby lifting the degeneracies of both hole and electron states. This interaction becomes stronger with decreasing QD size due to the increased overlap between hole and electron wave functions. ${ }^{7}$ Therefore, for small QDs shape asymmetry effects can be considered as a perturbation to the exchange interaction, while for larger QDs shape effects will give the dominating contribution. ${ }^{7}$ The band-edge exciton fine structure within the EMA framework is thus strongly size and shape dependent. For example, in the size range from 4.0 to $2.4 \mathrm{~nm}$ diameter $\Delta E$ is calculated to increase from 5.5 to $14.2 \mathrm{meV}$ for spherical QDs and from 0.6 to $5.5 \mathrm{meV}$ for prolate QDs with an aspect ratio of 1.28 . $^{7}$

In the many-body pseudopotential theory developed by Zunger et $a l .{ }^{9}$ crystal-field, spin-orbit coupling and shape effects are not treated perturbatively but instead are built at the outset into the exciton wave functions. The results obtained by this theoretical approach are similar to those calculated within the EMA framework, although the size dependence of the electron-hole exchange interaction is found to be smaller (i.e., $R^{-2}$ instead of $R^{-3}$ ), probably because correlation effects and the screening of the electronhole interaction by the dielectric constant were taken into account. ${ }^{9}$ A strongly size- and shape-dependent band-edge exciton fine structure was also obtained for $\mathrm{CdSe}$ nanocrystals within the semiempirical tight-binding framework used by Whaley et al. ${ }^{10}$ This theoretical model included crystal-field, electron-hole exchange, spin-orbit 
coupling, and configuration interactions, analyzing also the effects of shape and the discrete lattice structure. The nanocrystal is no longer modeled as an ellipsoid but more realistically as slightly prolate and facetted nanocrystals with truncated tops $\left(C_{3 v}\right.$ point symmetry $) .{ }^{10}$ The splitting of the exciton levels and the oscillator strengths of electronic transitions are shown to be sensitive to small structural perturbations such as shape changes ${ }^{10}$ and surface relaxation..$^{53}$ For example, increasing the aspect ratio of $2.8 \mathrm{~nm}$ QDs from 1.05 to 1.44 decreases the calculated $\Delta E$ from 14 to $3.9 \mathrm{meV} .{ }^{10} \mathrm{~A}$ similar spread in the $\Delta E$ values is also obtained by keeping the size and aspect ratio constant and varying the shape of the nanocrystal (e.g., from 12 to $5.4 \mathrm{meV}$ for $2.8 \mathrm{~nm}$ QDs with an aspect ratio of 1.1 but different geometrical shapes, i.e., facetted differently). ${ }^{10}$

It is thus clear that the theoretically calculated $\Delta E$ values are extremely sensitive to the size and shape of the QDs, ${ }^{7,9,10}$ implying that the discrepancy between the theoretically predicted $\Delta E$ values and those reported here may be due to shape effects, since the QDs investigated here are prolate (aspect ratio: $1.3 \pm 0.2$ ) and highly facetted CdSe nanocrystals, as shown by TEM and high-resolution TEM measurements. However, the theoretical values for CdSe QDs smaller than $3 \mathrm{~nm}$ [e.g., $3.9 \mathrm{meV}$ for a $2.8 \mathrm{~nm}$ prolate QD with aspect ratio of 1.28 (Ref. 7) or 1.44 (Ref. 10)] are still larger than those observed here [Fig. 6(b)], which suggests that there may be other mechanisms contributing to reduce the electron-hole exchange interaction.

Before analyzing possible alternative mechanisms we will discuss the temperature- and size-dependence of the exciton lifetimes. The low-temperature exciton lifetimes observed here are consistent with available experimental data, ${ }^{7,11-13,24,25}$ but much shorter than EMA-based theoretical predictions. ${ }^{7}$ The levels shown in Fig. 1 are labeled according to their total angular momentum projection, ${ }^{7}$ but they can also be classified according to their spin orientation as singlet (bright) and triplet (dark) states (e.g., the $E_{ \pm 1}^{L}$ and $E_{ \pm 2}$ levels, respectively).Within the EMA framework the dark exciton (triplet) lifetime should be size independent and infinite, ${ }^{7}$ and additional symmetry breaking mechanisms such as excitonphonon coupling have to be invoked to account for the finite microsecond lifetimes experimentally observed. ${ }^{7,11-13,24,25}$ However, semiempirical tight-binding calculations ${ }^{10}$ have successfully reconciled experiment and theory by showing that finite triplet (dark exciton) radiative lifetimes in the microsecond range can be obtained for CdSe QDs by incorporating interactions with higher exciton states and explicitly taking into account the atomic composition and geometry of the nanocrystal, which leads to mixing of singlet (bright exciton) character into nominally triplet (dark) states. ${ }^{10}$

The size-dependence observed here (Fig. 6) can be qualitatively understood in terms of size-dependent changes in the CdSe intrinsic band-edge exciton fine structure, despite the quantitative disagreement with the theoretical $\Delta E$ values (see discussion above). Reduction in the QD size increases the confinement energies and the electron-hole wave function overlap, thereby enhancing the electron-hole exchange interaction, ${ }^{7,9,13}$ resulting in an increase of the dark-bright (triplet-singlet) energy gap $\Delta E$ (Fig. 6). As mentioned above, semiempirical tight-binding calculations ${ }^{10}$ show that the mixing of a singlet character into triplet exciton states is expected to be inherent in CdSe QDs as a consequence of their chemical composition (i.e., the spin-orbit coupling is rather strong in $\mathrm{Cd}$ and $\mathrm{Se}$ ). Considering that the mixing between singlet and triplet states should be inversely proportional to the singlet-triplet energy gap, therefore increasing with decreasing $\Delta E$, one can conclude that the radiative lifetime of the dark exciton should decrease with increasing QD size, in agreement with the experimentally observed size dependence [Fig. 6(a)].

We will now examine whether alternative mechanisms can satisfactorily explain our observations. It is worth noting that the activation energies determined by Crooker et al. ${ }^{24}$ from the temperature dependence of the exciton lifetimes of $\mathrm{CdSe} / \mathrm{ZnS}$ QDs $(\sim 1 \mathrm{meV}$ from 2.6 to $4.2 \mathrm{~nm}$ diameter) are also much smaller than the reported $\Delta_{S T}$ values ${ }^{7,13}$ or the theoretically predicted $\Delta E$ 's, ${ }^{7,9,10}$ but are comparable to the $\Delta E$ values presented here (viz., 0.84 to $1.03 \mathrm{meV}$ from 4.3 to $2.5 \mathrm{~nm}$ diameter, see [Fig. 6(b)]). The fact that a sizedependence was not observed in Ref. 24 is probably due to the limited size range investigated $(2.6-4.2 \mathrm{~nm}$ diameter). The discrepancy between the experimental data and the EMA-based theoretical predictions was attributed in Ref. 24 to the presence of an additional recombination channel with $a \sim 1 \mathrm{meV}$ activation energy, common to all QDs, which was ascribed to a weak exchange interaction between the dark exciton and the ensemble of dangling bonds on the QD surface, resulting in a spin-flip assisted recombination directly from the $E_{ \pm 2}$ level. ${ }^{24} \mathrm{~A}$ similar surface-assisted recombination model (viz., interaction of photogenerated carriers with uncompensated spins at the QD surface) has been proposed by Johnston-Halperin et al. ${ }^{52}$ to explain the fact that the lowtemperature (below $4 \mathrm{~K}$ ) exciton $g$ factor extracted from polarization-resolved magnetophotoluminescence measurements showed an unexpected temperature behavior (decrease with decreasing temperature) and was much smaller than theoretically predicted within the EMA framework. The authors nevertheless stated that, apart from the inconsistency mentioned above, their results appeared to confirm the EMA-based theoretical predictions. ${ }^{52}$

The increase upon cooling of the relative intensity of the phonon replica lines in the FLN spectra (i.e., the increase of the Huang-Rhys parameter $S)^{12,13}$ has been taken as supporting evidence for both phonon- ${ }^{7,13}$ and surface-assisted ${ }^{12,24,51}$ radiative recombination of the dark exciton, since it suggests the existence of an activation energy for zero-phonon line emission. It has been recently argued ${ }^{24,51}$ that the observation of zero-phonon line emission at temperatures below $2 \mathrm{~K}$ (i.e., dark exciton luminescence) $)^{12,13}$ provides additional support for the surface-assisted model, based on the assumption that thermal population of the bright exciton and phonon assistance would be both negligible below $2 \mathrm{~K}$, and therefore an additional recombination channel for the dark exciton would be needed in order to explain the finite lifetimes observed ${ }^{24,51}$ However, as discussed above, finite microsecond lifetimes can also be obtained by taking singlet-triplet mixing into account. ${ }^{10}$ It is also interesting to note that the assignment of the low-temperature FLN spectra to surface states by Bawendi et al. ${ }^{12}$ was later dismissed by the same group for being inconsistent with transient differential 
absorption and gated FLN spectra ${ }^{32}$ and with a large body of experimental $7,13,33,34$ and theoretical work. 7,13 Further, the temperature (and size) dependence of the Huang-Rhys parameter $^{12}$ can easily be understood by assuming that the exciton-phonon coupling is larger for the dark exciton than for the bright exciton (the $E_{ \pm 2}$ and the $E_{ \pm 1}^{L}$ levels, respectively). This would also (qualitatively) explain the increase in the homogeneous exciton linewidth with decreasing QD size. Moreover, phonon-assistance is very effective in relaxing selection rules by symmetry breaking and/or mixing, 7,13 and will be active even at low temperatures for processes involving the creation of phonons [viz., Stokes vibronic replicas, such as those observed in the FLN spectra of CdSe QDs (Refs. 12 and 13)].

Recently published semiempirical pseudopotential calculations ${ }^{51}$ provided theoretical support for the idea that the dark exciton radiative recombination rates are determined by surface states, by showing that surface-localized hole-trap states can promote the mixing of dark and bright exciton states, therefore decreasing the dark exciton radiative lifetime from milliseconds to a microsecond. ${ }^{51}$ However, the calculations yield a size independent dark exciton radiative lifetime, ${ }^{51}$ which is not consistent with the clear size dependence observed here [Fig. 6(a)]. A similar, albeit less pronounced, size dependence for the exciton lifetimes below $2 \mathrm{~K}$ was also observed by Crooker et al. ${ }^{24}$ for $\mathrm{CdSe} / \mathrm{ZnS}$ QDs (1.13-0.87 $\mu$ s from 2.6 to $4.2 \mathrm{~nm}$ diameter). Furthermore, the exciton lifetimes at $1.3 \mathrm{~K}$ [Figs. 3 and 6(a)] and the $\Delta E$ values [Fig. 6(b)] for the $\mathrm{CdSe} / \mathrm{ZnSe} / \mathrm{ZnS}$ core-shellshell QDs and the original organically capped $2.8 \mathrm{~nm} \mathrm{CdSe}$ cores are observed to be the same, despite the strongly different surfaces. This shows that the dark exciton radiative lifetime and the dark-bright energy gap are not significantly affected by surface states. This conclusion is further supported by the absence of photoluminescence recovery (LTAQ, regime III in Fig. 4) for core-shell QDs, since this demonstrates the ability of high-quality inorganic shells to shield the QD core from surface perturbations. Also, the similarity between the results presented here and in Ref. 24 strongly argues against the involvement of surface states, since it is unlikely that systems with different surfaces and/or interfaces, such as the samples investigated in the present work (viz., CdSe/ZnSe/ZnS core-shell QDs and HDATOPO capped CdSe QDs) and in Ref. 24 (CdSe/ZnS coreshell QDs and Cd-rich TOPO-TOP capped CdSe QDs), would have similar surface and/or interface states, so that comparable activation energies (i.e., $\sim 1 \mathrm{meV}$ ) would be observed. These observations provide compelling evidence that the observed exciton PL decay is due to a mechanism intrinsic to the CdSe QDs, even at the lowest temperatures investigated.
The investigation of the two-photon excitation spectra of CdSe QDs (Ref. 54) has provided evidence that the current understanding of the exciton fine structure of QDs still requires refining. Although the observation of the formally parity-forbidden $1 S_{3 / 2(h)} \rightarrow 1 S_{(e)}$ transition can be understood by taking into account the QD shape (prolate and facetted, $C_{3 v}$ point group symmetry, thus lacking inversion symmetry), the absence of transitions involving the $E_{ \pm 2}$ level (i.e., the dark exciton) is intriguing. ${ }^{54}$ Based on these observations it has been suggested that the current theoretical descriptions of the electronic fine structure of CdSe QDs should be revised in order to include the effect of permanent dipole moments. ${ }^{54}$ The existence of a permanent dipole moment in $\mathrm{CdSe}$ nanocrystals (and nanorods) has been recently demonstrated ${ }^{55,56}$ and shown to be intrinsic to the wurtzite lattice and the asymmetric shapes of CdSe nanocrystals. ${ }^{56}$ The presence of a permanent dipole moment could lead to additional mixing of hole states (e.g., $1 S_{3 / 2(h)}$ and $1 P_{3 / 2(h)}$ ), therefore altering the magnitude of the electron-hole exchange interaction and the mixing of spin states. ${ }^{54}$ However, it is as yet unclear to what extent a permanent dipole moment (or other additional mechanisms) would change the bandedge exciton fine structure, and further experiments will be needed in order to elucidate this issue.

\section{CONCLUSIONS}

We have investigated the size- and temperaturedependence of exciton lifetimes in CdSe QDs. Three temperature regimes can be recognized, regardless of the QD size. The low-temperature regime (below $\sim 50 \mathrm{~K}$ ) is characterized by purely radiative decays and strong size- and temperature-dependencies, resulting in longer lifetimes at lower temperatures and for smaller QDs. The intermediate temperature regime $(\sim 50-250 \mathrm{~K})$ is governed by thermally activated quenching. The high-temperature regime (above $\sim 250 \mathrm{~K}$ ) is characterized by decreasing nonradiative decay rates with the consequent recovery of the PL quantum yields ("luminescence temperature antiquenching"). This regime is strongly dependent on the surfactant shell and is essentially size independent. The size- and temperaturedependencies observed in the radiative regime (below $\sim 50 \mathrm{~K}$ ) can be understood in terms of the CdSe band-edge exciton fine structure and modeled by a thermal distribution between a lower dark (triplet) and a higher bright (singlet) exciton state. The bright-dark energy gap $(\Delta E)$ and the dark (triplet) exciton lifetime increase with decreasing QD size, as a result of the increase of the confinement energies and stronger electron-hole exchange interaction.
*Corresponding author. Fax: +31-30-2532403; Email address:
C.deMelloDonega @ phys.uu.nl
${ }^{1}$ For recent reviews see C. Burda, X. Chen, R. Narayanan, and M.
A. El-Sayed, Chem. Rev. (Washington, D.C.) 105, 1025 (2005);
C. de Mello Donegá, P. Liljeroth, and D. Vanmaekelbergh, Small 1, 664 (2005).

${ }^{2}$ R. A. M. Hikmet, P. T. K. Chin, D. V. Talapin, and H. Weller, Adv. Mater. (Weinheim, Ger.) 17, 1436 (2005). 
${ }^{3}$ I. Gur, N. A. Fromer, M. L. Geier, and A. P. Alivisatos, Science 310, 462 (2005).

${ }^{4}$ X. Michalet, F. F. Pinaud, L. A. Bentolila, J. M. Tsay, S. Doose, J. J. Li, G. Sundaresan, A. M. Wu, S. S. Gambhir, and S. Weiss, Science 307, 538 (2005).

${ }^{5}$ W. J. M. Mulder, R. Koole, R. J. Brandwijk, G. Storm, P. T. K. Chin, G. J. Strijkers, C. de Mello Donegá, K. Nicolay, and A. W. Griffioen, Nano Lett. 6, 1 (2006).

${ }^{6}$ D. V. Talapin and C. B. Murray, Science 310, 86 (2005).

${ }^{7}$ Al. L. Efros, M. Rosen, M. Kuno, M. Nirmal, D. J. Norris, and M. Bawendi, Phys. Rev. B 54, 4843 (1996).

${ }^{8}$ Al. L. Efros and M. Rosen, Annu. Rev. Mater. Sci. 30, 475 (2000).

${ }^{9}$ A. Franceschetti, H. Fu, L. W. Wang, and A. Zunger, Phys. Rev. B 60, 1819 (1999).

${ }^{10}$ K. Leung, S. Pokrant, and K. B. Whaley, Phys. Rev. B 57, 12291 (1998).

${ }^{11}$ M. G. Bawendi, P. J. Carroll, W. L. Wilson, and L. E. Brus, J. Chem. Phys. 96, 946 (1992).

${ }^{12}$ M. Nirmal, C. B. Murray, and M. G. Bawendi, Phys. Rev. B 50, 2293 (1994).

${ }^{13}$ M. Nirmal, D. J. Norris, M. Kuno, M. G. Bawendi, Al. L. Efros, and M. Rosen, Phys. Rev. Lett. 75, 3728 (1995).

${ }^{14}$ P. Guyot-Sionnest, B. Wehrenberg, and D. Yu, J. Chem. Phys. 123, 074709 (2005).

${ }^{15}$ S. Xu, A. A. Mikhailovsky, J. A. Hollingsworth, and V. I. Klimov, Phys. Rev. B 65, 045319 (2002).

${ }^{16}$ E. Hendry, M. Koeberg, F. Wang, H. Zhang, C. de Mello Donegá, D. Vanmaekelbergh, and M. Bonn, Phys. Rev. Lett. 96, 057408 (2006).

${ }^{17}$ H. Wang, C. de Mello Donegá, A. Meijerink, and M. Glassbeek, J. Phys. Chem. B 110, 733 (2006).

${ }^{18}$ A. M. Kapitonov, A. P. Stupak, S. V. Gaponenko, E. P. Petrov, A. L. Rogach, and A. Eychmüller, J. Phys. Chem. B 103, 10109 (1999).

${ }^{19}$ G. Schlegel, J. Bohnenberger, I. Potapova, and A. Mews, Phys. Rev. Lett. 88, 137401 (2002).

${ }^{20}$ X.-J. Wang, J.-Y. Zhang, A. Nazzal, M. Darragh, and M. Xiao, Appl. Phys. Lett. 81, 4829 (2002).

${ }^{21}$ C. de Mello Donegá, S. G. Hickey, S. F. Wuister, D. Vanmaekelbergh, and A. Meijerink, J. Phys. Chem. B 107, 489 (2003).

${ }^{22}$ X. Wang, L. Qu, J. Zhang, X. Peng, and M. Xiao, Nano Lett. 3, 1103 (2003).

${ }^{23}$ A. Javier, D. Magana, T. Jennings, and G. F. Strouse, Appl. Phys. Lett. 83, 1423 (2003).

${ }^{24}$ S. A. Crooker, T. Barrick, J. A. Hollingsworth, and V. I. Klimov, Appl. Phys. Lett. 82, 2793 (2003).

${ }^{25}$ O. Labeau, P. Tamarat, and B. Lounis, Phys. Rev. Lett. 90, 257404 (2003).

${ }^{26}$ B. R. Fisher, H.-J. Eisler, N. E. Stott, and M. G. Bawendi, J. Phys. Chem. B 108, 143 (2004).

${ }^{27}$ S. F. Wuister, C. de Mello Donegá, and A. Meijerink, J. Chem. Phys. 121, 4310 (2004).

${ }^{28}$ A. F. van Driel, G. Allan, C. Delerue, P. Lodahl, W. L. Vos, and D. Vanmaekelbergh, Phys. Rev. Lett. 95, 236804 (2005).

${ }^{29}$ S. F. Wuister, C. de Mello Donegá, and A. Meijerink, J. Am.
Chem. Soc. 126, 10397 (2004).

${ }^{30}$ S. F. Wuister, A. van Houselt, C. de Mello Donegá, D. Vanmaekelbergh, and A. Meijerink, Angew. Chem., Int. Ed. 43, 3029 (2004).

${ }^{31}$ S. S. Prabhu, A. S. Vengurlekar, S. K. Roy, and J. Shabb, Phys. Rev. B 50, 18098 (1994).

${ }^{32}$ D. J. Norris and M. G. Bawendi, J. Chem. Phys. 103, 5260 (1995).

${ }^{33}$ M. Kuno, J. K. Lee, B. O. Dabbousi, F. V. Mikulec, and M. G. Bawendi, J. Chem. Phys. 106, 9869 (1997).

${ }^{34}$ D. J. Norris, Al. L. Efros, M. Rosen, and M. G. Bawendi, Phys. Rev. B 53, 16347 (1996).

${ }^{35}$ L. Qu and X. Peng, J. Am. Chem. Soc. 124, 2049 (2002).

${ }^{36}$ D. V. Talapin, I. Mekis, S. Gotzinger, A. Kornowski, O. Benson, and H. Weller, J. Phys. Chem. B 108, 18826 (2004).

${ }^{37}$ R. Xie, U. Kolb, J. Li, T. Basché, and A. Mews, J. Am. Chem. Soc. 127, 7480 (2005).

${ }^{38}$ P. Reiss, J. Bleuse, and A. Pron, Nano Lett. 2, 781 (2002).

${ }^{39}$ J. S. Steckel, J. P. Zimmer, S. Coe-Sullivan, N. E. Stott, V. Bulovic, and M. G. Bawendi, Angew. Chem., Int. Ed. 43, 2154 (2004).

${ }^{40}$ W. William Yu, L. Qu, W. Guo, and X. Peng, Chem. Mater. 15, 2854 (2003).

${ }^{41}$ C. A. Leatherdale, W.-K. Woo, F. V. Mikulec, and M. G. Bawendi, J. Phys. Chem. B 106, 7619 (2002).

${ }^{42}$ C. B. Murray, D. J. Norris, and M. G. Bawendi, J. Am. Chem. Soc. 115, 8706 (1993).

${ }^{43}$ M. Jacobsohn and U. Banin, J. Phys. Chem. B 104, 1 (2000).

${ }^{44}$ V. N. Soloviev, A. Eichhofer, D. Fenske, and U. Banin, J. Am. Chem. Soc. 115, 8706 (2000).

${ }^{45}$ X. Peng, J. Wickham, and A. P. Alivisatos, J. Am. Chem. Soc. 120, 5343 (1998).

${ }^{46}$ D. Válerini, A. Creti, M. Lomascolo, L. Manna, R. Cingolani, and M. Anni, Phys. Rev. B 71, 235409 (2005).

${ }^{47}$ I. Broser, R. Broser, and A. Hoffman, in Physics of Group II-IV Elements and I-VII, edited by O. Madelung, M. Schultz, and H. Weiss, Landolt Börnstein New series, Group III, Vol. 17, Pt. B (Springer-Verlag, Berlin, 1988), p. 202.

${ }^{48}$ G. W. Walker, V. C. Sundar, C. M. Rudzinski, A. W. Wun, M. G. Bawendi, and D. G. Nocera, Appl. Phys. Lett. 83, 3555 (2003).

${ }^{49}$ E. W. J. L. Oomen, G. J. Dirksen, W. M. A. Smit, and G. Blasse, J. Phys. C 20, 1161 (1987).

${ }^{50}$ Y. Ebenstein, T. Makari, and U. Banin, Appl. Phys. Lett. 80, 4033 (2002).

${ }^{51}$ M. Califano, A. Franceschetti, and A. Zunger, Nano Lett. 5, 2360 (2005).

${ }^{52}$ E. Johnston-Halperin, D. D. Awschalom, S. A. Crooker, Al. L. Efros, M. Rosen, X. Peng, and A. P. Alivisatos, Phys. Rev. B 63, 205309 (2001).

${ }^{53}$ K. Leung and K. B. Whaley, J. Chem. Phys. 110, 11012 (1999).

${ }^{54}$ M. E. Schmidt, S. A. Blanton, M. A. Hines, and P. GuyotSionnest, J. Chem. Phys. 106, 5254 (1997).

${ }^{55}$ M. Shim and P. Guyot-Sionnest, J. Chem. Phys. 111, 6955 (1999).

${ }^{56}$ L. S. Li and A. P. Alivisatos, Phys. Rev. Lett. 90, 097402 (2003). 